\title{
Article
}

\section{Pseudo-commutative algebras}

\author{
Hee Sik Kim ${ }^{1}$, J. Neggers ${ }^{2}$ and Sun Shin Ahn ${ }^{3, *}$ \\ ${ }^{1}$ Department of Mathematics, Research Institute of Natural Sciences, Hanyang University, Seoul 04763, Korea; \\ heekim@hanyang.ac.kr \\ ${ }^{2}$ Department of Mathematics, University of Alabama, Tuscaloosa, AL 35487-0350, U. S. A.; jneggers@ua.edu \\ ${ }^{3}$ Sun Shin Ahn, Department of Mathematics Education, Dongguk University, Seoul 04620, Korea; \\ sunshine@dongguk.edu \\ *Correspondences: sunshine@dongguk.edu, mobile: +82-10-3783-3410; office: +82-2-2260-3410.
}

Received: 14 October 2018;

\begin{abstract}
In this paper, we investigate the effects of certain variants of the commutative laws on properties of several families of algebras which are in general not commutative, such as groups, linear algebras, or actually quite anti-commutative, such as $B C K$-algebras and $d$-algebras among others. From results obtained it becomes clear that by considering these variants in the presence of yet other axioms it is to be expected that a quite rich and varied set of results may be obtained both in the general and the particular setting of which what has been accomplished in this paper is a substantial sample.
\end{abstract}

Keywords: (left-, right-) pseudo-commutative, commutative, $d / B C K$-algebra, Smarandache disjoint, (linear) groupoid.

\section{Introduction}

If $(X, *)$ is a particular type of groupoid (binary system) on a set $X$, then one is often interested in observing how properties or axioms are maintained in closely related groupoids. Such is the case when we are dealing with homomorphic images and isotopies for example ([3]). In the following paper the "deformation" in question is a deformation of the commutativity axiom $x * y=y * x$ to one of several different forms. In particular, in highly non-commutative systems such as $B C K$-algebras and $d$-algebras, for example, such considerations are often helpful in obtaining conditions on such algebras which imply that such pseudo-commutativity conditions are indeed of interest and their implications in different settings worth investigating.

\section{Preliminaries}

A d-algebra ([8]) is a non-empty set $X$ with a constant 0 and a binary operation " * " satisfying the following axioms: (I) $x * x=0$, (II) $0 * x=0$, (III) $x * y=0$ and $y * x=0$ imply $x=y$, for all $x, y \in X$. For more detailed information we refer to $[2,4,5,7]$.

A $B C K$-algebra ([6]) is a $d$-algebra $X$ satisfying the following additional axioms: (IV) $((x * y) *$ $(x * z)) *(z * y)=0,(\mathrm{~V})(x *(x * y)) * y=0$ for all $x, y, z \in X$.

A $d$ / BCK-algebra $(X, *, 0)$ is said to be bounded if there exists an element $1 \in X$ such that $x * 1=0$ for any $x \in X$. A $d / B C K$-algebra $(X, *, 0)$ is said to be commutative if $x *(x * y)=y *(y * x)$ for all $x, y \in X$, i.e., $x \wedge y=y \wedge x$.

Theorem 1. ([6]) For a bounded commutative BCK-algebra $(X, *, 0)$, we have

(i) $N N x=x$ for all $x \in X$,

(ii) $N x \vee N y=N(x \wedge y), N x \wedge N y=N(x \vee y)$ for all $x, y \in X$,

(iii) $N x * N y=y * x$ for all $x, y \in X$. 


\section{Pseudo commutative algebras}

Given a groupoid (or an algebra) $(X, *)$, it is said to be

(i) commutative if $x * y=y * x$;

(ii) left-pseudo-commutative if $x * y=\varphi(y) * x$ for some map $\varphi: X \rightarrow X$;

(iii) right-pseudo-commutative if $x * y=y * \varphi(x)$ for some map $\varphi: X \rightarrow X$;

(iv) pseudo-commutative if $x * y=\varphi(y) * \varphi(x)$ for some map $\varphi: X \rightarrow X$,

for all $x, y \in X$.

Example 1. Let $\mathbf{N}:=\{0,1,2, \cdots\}$ be a set. If we define a binary operation " $*$ " on $\mathbf{N}$ by $m * n:=(n+1) * m$, then $\varphi(n)=n+1$ yields $m * n=\varphi(n) * m$ and $(\mathbf{N}, *)$ is left-pseudo-commutative. Assume that $(\mathbf{N}, *)$ is left-pseudo-commutative. Let us consider the orbits generated

$$
\begin{aligned}
& 0:=0 * 0=1 * 0=1 * 1=2 * 1=2 * 2=3 * 2=\cdots \\
& 1:=0 * 1=2 * 0=1 * 2=3 * 1=2 * 3=4 * 2=\cdots \\
& 2:=0 * 2=3 * 0=1 * 3=4 * 1=2 * 4=5 * 2=\cdots \\
& k:=0 * k=(k+1) * 0=1 *(k+1)=\cdots
\end{aligned}
$$

Assume that $(\mathbf{N}, *)$ is right-pseudo-commutative. Then $m * n=n * \psi(m)$ for some map $\psi: \mathbf{N} \rightarrow \mathbf{N}$. Then $0 * 0=0 * \psi(0)=0$ and $\psi(0)=0$ by the above table. Also $1=0 * 1=1 * \psi(0)=1 * 0=0$, a contradiction. Hence $(\mathbf{N}, *)$ is not right-pseudo-commutative. Next, suppose that $m * n:=\xi(n) * \xi(m)$ for some map $\xi: \mathbf{N} \rightarrow \mathbf{N}$. Then $0=1 * 0=\xi(0) * \xi(1)$ and hence $\xi(0)=\xi(1)$ or $\xi(0)=\xi(1)+1$. If $\xi(0)=\xi(1)$, then $1=0 * 1=\xi(1) * \xi(0)=0$, a contradiction. If $\xi(0)=\xi(1)+1$, then $1=0 * 1=\xi(1) * \xi(0)=$ $\xi(1) *(\xi(1)+1) .2=0 * 2=\xi(2) * \xi(0)$ and so $\xi(0)=\xi(2)+2$ or $\xi(2)=\xi(0)+3$ by the above table. $1=2 * 0=\xi(0) * \xi(2)$. If $\xi(0)=\xi(2)+2$, then $(\xi(2)+2) * \xi(2)=1$ is possible and $\xi(0) *(\xi(0)+3)=3$ is impossible. Thus $\xi(0)=\xi(1)+1=\xi(2)+2=\xi(3)+3=\cdots$ and $\xi(0) \geq n$ for all $n$, a contradiction.

Note that if we define $m * n:=n *(m+1)$ on $(\mathbf{N}, *)$, then we can easily construct an example of right-pseudo-commutative algebra which is neither left-pseudo-commutative nor pseudo-commutative.

Example 2. Let $X:=\{0,1,2,3\}$ be a set. If we define a map $\varphi: X \rightarrow X$ by $\varphi(0)=0, \varphi(1)=2, \varphi(2)=3$ and $\varphi(3)=1$, then the algebra(groupoid) $(X, *)$ with the following table is left-pseudo-commutative, but not commutative.

\begin{tabular}{l|llll}
$*$ & 0 & 1 & 2 & 3 \\
\hline 0 & $\alpha$ & $\beta$ & $\beta$ & $\beta$ \\
1 & $\beta$ & $\gamma$ & $\delta$ & $\gamma$ \\
2 & $\beta$ & $\gamma$ & $\gamma$ & $\delta$ \\
3 & $\beta$ & $\delta$ & $\gamma$ & $\gamma$
\end{tabular}


where $\alpha, \beta, \gamma, \delta$ are distinct elements of $X$. For example, if we let $\alpha=0, \beta=2, \gamma=1, \delta=3$, then the algebra $(X, *)$ with the following table is left-pseudo-commutative, but not commutative.

\begin{tabular}{l|llll}
$*$ & 0 & 1 & 2 & 3 \\
\hline 0 & 0 & 2 & 2 & 2 \\
1 & 2 & 1 & 3 & 1 \\
2 & 2 & 1 & 1 & 3 \\
3 & 2 & 3 & 1 & 1
\end{tabular}

Example 3. Given a set $X:=\{0,1,2,3\}$ with a map $\varphi$ defined in Example 2, the algebra $(X, *)$ with the following table is right-pseudo-commutative, but not commutative, since $1 * 2=\gamma$ and $\varphi(2) * \varphi(1)=3 * 2=$ $\delta$.

\begin{tabular}{l|llll}
$*$ & 0 & 1 & 2 & 3 \\
\hline 0 & $\alpha$ & $\beta$ & $\beta$ & $\beta$ \\
1 & $\beta$ & $\gamma$ & $\gamma$ & $\delta$ \\
2 & $\beta$ & $\delta$ & $\gamma$ & $\gamma$ \\
3 & $\beta$ & $\gamma$ & $\delta$ & $\gamma$
\end{tabular}

where $\alpha, \beta, \gamma, \delta$ are distinct elements of $X$. For example, the algebra $(X, *)$ with the following table is right-pseudo-commutative, but not commutative.

\begin{tabular}{l|llll}
$*$ & 0 & 1 & 2 & 3 \\
\hline 0 & 0 & 1 & 1 & 1 \\
1 & 1 & 2 & 2 & 3 \\
2 & 1 & 3 & 2 & 2 \\
3 & 1 & 2 & 3 & 2
\end{tabular}

Proposition 1. If $(X, *)$ is commutative, then it is (left-, right-) pseudo-commutative.

Proof. Take $\varphi:=i d_{X}$.

Given algebra types $(X, *)$ (type- $\left.P_{1}\right)$ and $(X, \circ)$ (type- $\left.P_{2}\right)$, we shall consider them to be Smarandache disjoint ([1]) if the following two conditions hold:

(i) If $(X, *)$ is a type- $P_{1}$-algebra with $|X|>1$ then it cannot be a Smarandache-type- $P_{2}$-algebra $(X, \circ)$;

(ii) If $(X, \circ)$ is a type- $P_{2}$-algebra with $|X|>1$ then it cannot be a Smarandache-type- $P_{1}$-algebra $(X, *)$.

A groupoid $(X, *)$ is said to be a left-zero-semigroup if $x * y=x$ for any $x, y \in X$.

Proposition 2. Left-zero-semigroups and (left-, right-) pseudo-commutative algebras are Smarandache disjoint.

Proof. Assume the left-zero-semigroup $(X, *)$ is left-pseudo- commutative. Then $x=x * y=\varphi(y) *$ $x=\varphi(y)$ for any $x, y \in X$ for some map $\varphi: X \rightarrow X$, which implies $\varphi(y)=x$ for all $x, y \in X$, and thus $|X|=1$.

Assume the left-zero-semigroup $(X, *)$ is right-pseudo-commutative. Then $x=x * y=y * \varphi(x)=$ $y$ for any $x, y \in X$ for some map $\varphi: X \rightarrow X$, and thus $|X|=1$.

Assume the left-zero-semigroup $(X, *)$ is pseudo-commutative. Then $x=x * y=\varphi(y) * \varphi(x)=$ $\varphi(y)$ for any $x, y \in X$ for some map $\varphi: X \rightarrow X$, which implies $\varphi(y)=x$ for all $x, y \in X$, and thus $|X|=1$.

Theorem 2. If the groupoid $(X, *)$ is a group, then (left-, right-) pseudo-commutativity implies commutativity. 
Proof. Let $(X, *, e)$ be a group which is left-pseudo-commutative. then $x * y=\psi(x) * x$ for some map $\psi: X \rightarrow X$, and so $x=x * e=\psi(e) * x$, so that $\psi(e)=e$. Also, $e=x * x^{-1}=\psi\left(x^{-1}\right) * x$ and $\psi\left(x^{-1}\right)=x^{-1}$, i.e., $\psi(x)=x$, so that $x * y=y * x$ and $(X, *, e)$ is commutative.

If $(X, *, e)$ is a group which is right-pseudo-commutative, then $x * y=y * \varphi(x)$ for some $\varphi: X \rightarrow$ $X$. Hence $x=x * e=e * \varphi(x)=\varphi(x)$ and $\varphi(x)=x$, so that $x * y=y * x$ and $(X, *, e)$ is commutative.

If $(X, *, e)$ is a group which is pseudo-commutative, then $e=x * x^{-1}=\xi\left(x^{-1}\right) * \xi(x)$ for some $\varphi: X \rightarrow X$, so that $\xi(x)^{-1}=\xi\left(x^{-1}\right)$. Hence $(\xi(e))^{-1}=\xi\left(e^{-1}\right)=\xi(e)$. Since $x=x * e=\xi(e) * \xi(x)=$ $(\xi(e))^{-1} * \xi(x)$, we obtain $\xi(e) * x=\xi(e) *\left[\left(\xi(e)^{-1} * \xi(x)\right]=\left[\xi(e) *(\xi(e))^{-1}\right] * \xi(x)=e * \xi(x)=\xi(x)\right.$. Let $a:=\xi(e)$. Then $a^{2}=e$ and $(a * y) *(a * x)=(\xi(e) * y) *(\xi(e) * x)=\xi(y) * \xi(x)=x * y$. Thus $a * x=(a * y)^{-1} *(x * y)=y^{-1} * a^{-1} * x * y=y^{-1} *(a * x) * y$. Since $x$ is arbitrary, $a * x$ is arbitrary, i.e., $a * x=u$ implies $y^{-1} * u * y=u * y$, i.e., $y * u=u * y$, i.e., $(X, *, e)$ is commutative.

Let $K$ be a field. A groupoid $(K, *)$ is said to be linear if $x * y:=A+B x+C y$ for any $x, y \in K$, where $A, B, C$ are (fixed) elements of $K$.

Proposition 3. If a linear groupoid $(K, *)$ is left-pseudo-commutative, then it is commutative.

Proof. Since $(K, *)$ is left-pseudo-commutative, there exists a map $\varphi: X \rightarrow X$ such that $x * y=\varphi(y) * x$ for any $x, y \in K$, i.e.,

$$
A+B x+C y=A+B \varphi(y)+C x
$$

If we let $x:=0, y:=1$ in (1) consequently, then $A+C y=A+B \varphi(y)$ and $C=B \varphi(1)$. If $B=C=0$, then $(K, *)$ is trivially commutative. If $B \neq 0$, then $\varphi(y)=\frac{C}{B} y$. By (1) we have $A+B x+C y=$ $A+C y+C x$, proving $B=C$. Thus, $x * y=A+B(x+y)=y * x$. Similarly, if $C \neq 0$, then $B=C$, proving $(K, *)$ is commutative.

Proposition 4. If a linear groupoid $(K, *)$ is right-pseudo-commutative, then it is commutative.

Proof. The proof is similar to Proposition 3, and we omit it.

Note that, in a linear groupoid $(K, *)$, the "pseudo-commutativity" does not imply the "commutativity". In fact, if we define $x * y:=A+B(x-y)$ for any $x, y \in K$, where $A, B \neq 0$ in $K$, then it is pseudo-commutative, but not commutative.

Proposition 5. Left-pseudo-commutative algebras and d-algebras are Smarandache disjoint.

Proof. Let $(X, *, 0)$ be a left-pseudo-commutative $d$-algebra. Then $x * y=\varphi(y) * x$ for any $x, y \in X$ for some map $\varphi: X \rightarrow X$. If we let $x:=0$, then $0=0 * y=\varphi(y) * 0$. Since $(X, *, 0)$ is $d$-algebra, by applying (I), (II), we obtain $\varphi(y)=0$ for any $y \in X$. Hence $x * y=\varphi(y) * x=0 * x=0$ for any $x, y \in X$, which implies $|X|=1$ by (III), proving the proposition.

Proposition 6. Non-bounded d-algebras and right-pseudo-commutative algebras are Smarandache disjoint.

Proof. Let $(X, *, 0)$ be a non-bounded right-pseudo-commutative $d$-algebras. Then there exists a map $\varphi: X \rightarrow X$ such that

$$
x * y=y * \varphi(x)
$$

for any $x, y \in X$. If we let $x:=0$ in (2), then $0=0 * y=y * \varphi(0)$ for any $y \in X$. We claim that $\varphi(0)=0$. In fact, if $\varphi(0) \neq 0$, then $y \leq \varphi(0)$ for any $y \in X$, i.e., $X$ is bounded, a contradiction. Hence $0=0 * y=y * \varphi(0)=y * 0$ for any $y \in X$, which means that $y=0$ for any $y \in X$, i.e., $|X|=1$. 
Note that, in a bounded $d$-algebra $(X, *, 0)$, if it is right-pseudo-commutative, then $x * y=y * \varphi(x)$ for any $x, y \in X$ for some map $\varphi: X \rightarrow X$. In this case, the map $\varphi$ is order-preserving. In fact, if $x * y=0$ in $X$, i.e., $x \leq y$, then $y * \varphi(x)=0$ and $\varphi(x) * \varphi(y)=0$, i.e., $\varphi(x) \leq \varphi(y)$.

Proposition 7. Let K be a field. Define a binary operation "*” on X by

$$
x * y:=x(x-y), \forall x, y \in K
$$

If $(K, *)$ is pseudo-commutative, then $K \cong Z_{2}=\{0,1\}$.

Proof. It is easy to show that $(K, *, 0)$ is a $d$-algebra. Assume that $(K, *)$ is pseudo-commutative. Then there exists a map $\varphi: K \rightarrow K$ such that $x * y=\varphi(y) * \varphi(x)$ for any $x, y \in K$. It follows that

$$
x * y=\varphi(y) * \varphi(x)=\varphi(y)[\varphi(y)-\varphi(x)]
$$

If we let $x:=0$ in (3), then $0=0 * y=\varphi(y)[\varphi(y)-\varphi(0)]$. Assume that $\varphi(y) \neq 0$. Then $\varphi(0)=$ $\varphi(y) \neq 0$, i.e., $\varphi(0) \neq 0$, say $\varphi(0)=u$. Hence $\varphi(y)=u, \forall y \in X$. Thus, we have either $\varphi(y)=0$ or $\varphi(y)=u \neq 0$, for all $y \in K$. This means that $|\varphi(K)| \leq 2$ and $|\varphi(K) \times \varphi(K)| \leq 4$. Since $(X, *)$ is pseudo-commutative, $x * y=\varphi(y) * \varphi(x)$ has at most 4 elements, i.e., $|K \times K| \leq 4$. Thus $K=K_{2}=$ $\{0,1\}$ is a possible field. If we let $\varphi(0):=1, \varphi(1):=0$, then $(K, *)$ is pseudo-commutative.

\section{4. $B C K$-algebras and a pseudo-commutativity}

Proposition 8. If a BCK-algebra $(X, *, 0)$ is pseudo-commutative, then it is bounded.

Proof. Let a $B C K$-algebra $(X, *, 0)$ be pseudo-commutative. Then $x * y=\varphi(y) * \varphi(x)$ for any $x, y \in X$ for some $\operatorname{map} \varphi: X \rightarrow X$. Since $0 * x=0, \forall x \in X, 0=\varphi(x) * \varphi(0), \forall x \in X$. This means that $\varphi(0)$ is the greatest element of $\varphi(X)$. We claim that $\varphi(0)$ is the greatest element of $X$. If we let $\alpha:=\varphi(0)$, since $x * 0=x$, we have $x=\varphi(0) * \varphi(x)=\alpha * \varphi(x), \forall x \in X$. Thus $x * \alpha=(\alpha * \varphi(x)) * \alpha=(\alpha * \alpha) * \varphi(x)=$ $0 * \varphi(x)=0, \forall x \in X$. Hence $\alpha=\varphi(0)$ is the greatest element of $X$, proving that $X$ is bounded.

The converse of Proposition 8 need not be true in general. See the following example.

Example 4. Consider a $B C K$-algebra $X:=\{0,1,2\}$ with the following table:

\begin{tabular}{l|lll}
$*$ & 0 & 1 & 2 \\
\hline 0 & 0 & 0 & 0 \\
1 & 1 & 0 & 0 \\
2 & 2 & 2 & 0
\end{tabular}

Then $(X, *, 0)$ is a bounded (positive-implicative) BCK-algebra (see [6, p. 243]). By routine calculations we see that there is no map $\varphi: X \rightarrow X$ satisfying the condition of a pseudo-commutativity, i.e., $(X, *, 0)$ is a bounded $B C K$-algebra having no pseudo-commutativity property.

The converse of Proposition 8 is also true if we add the condition of "commutativity".

Proposition 9. Every bounded commutative BCK-algebra is pseudo-commutative.

Proof. If we let $N x:=1 * x, \forall x \in X$, then, by Theorem 1-(iii), $x * y=N y * N x$ for all $x, y \in X$, proving that $(X, *, 0)$ is pseudo-commutative.

Proposition 10. If $(X, *, 0)$ is a pseudo-commutative BCK-algebra, then 
(i) $x=1 * \varphi(x), \forall x \in X$,

(ii) $\varphi(x) * \varphi(y)=(1 * \varphi(y)) *(1 * \varphi(x))$.

for some map $\varphi: X \rightarrow X$.

Proof. Since $X$ is pseudo-commutative, by Proposition $8, X$ is bounded and $\varphi(0)$ is the greatest element of $X$, say $1:=\varphi(0)$. Since $x * 0=x, \forall x \in X$, we obtain $x=x * 0=\varphi(0) * \varphi(x)=1 * \varphi(x), \forall x \in X$, proving (i). It follows from $X$ is pseudo-commutative that $\varphi(x) * \varphi(y)=y * x=(1 * \varphi(y)) *(1 * \varphi(x))$ by (i).

Theorem 3. Let $(X, *, 0)$ be a bounded commutative BCK-algebra. If a map $\varphi: X \rightarrow X$ satisfies the condition $x * y=\varphi(y) * \varphi(x), \forall x, y \in X$, then $\varphi(x)=N x, \forall x \in X$.

Proof. If $(X, *, 0)$ is a bounded commutative $B C K$-algebra, then by Proposition 9, it is pseudo-commutative and hence $x * y=\varphi(y) * \varphi(x), \forall x, y \in X$. By Proposition 10-(i), we have $x=1 * \varphi(x)$. Hence $N x=1 * x=1 *(1 * \varphi(x))=\varphi(x) *(\varphi(x) * 1)=\varphi(x) * 0=\varphi(x), \forall x \in X$, since $(X, *, 0)$ is commutative. This proves the theorem.

\section{Acknowledgments:}

Conflicts of Interest: The author declares no conflicts of interest.

\section{Referneces}

1. Allen, P. J.; Kim, H. S.; Neggers, J. Smarandache disjoint in BCK/d-algebras, Sci. Math. Jpn 2005 61, 447-449.

2. Allen, P. J.; Kim, H. S.; Neggers, J. On companion algebras, Math. Slovaca 2007 57, 93-106.

3. Bruck, R. H. A survey of binary systems, Springer-Verlag, New York, 1971.

4. Jun, Y. B.; Neggers, J.; Kim, H. J. Fuzzy $d$-ideals of $d$-algebras, J. Fuzzy Math. 2000 8, 123-130.

5. Lee, Y. C.; Kim, H. S. On $d$-subalgebras of $d$-transitive $d^{*}$-algebras, Math. Slovaca 1999 49, 27-33.

6. Meng, J.; Jun, Y. B. BCK-algebras, Kyung Moon Sa, Seoul, 1994.

7. Neggers,J.; Jun, Y. B.; Kim, H. S. On d-ideals in d-algebras, Math. Slovaca 1999 49, $243-251$.

8. Neggers J.; Kim, H. S. On d-algebras, Math. Slovaca 1999 49, 19-26.

9. Tanaka, S. Examples of BCK-algebras, Math. Seminar Notes 1975 3, 75-82. 